Kompass

Autoimmun

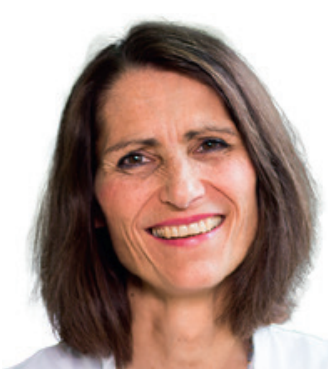

\title{
Rücken und Schmerz: Nicht immer nur entzündlich!
}

\author{
Sabine Adler
}

Fachbereich Gastroenterologie/Hepatologie, Endokrinologie/Diabetologie, Rheumatologie, Helios Klinikum Erfurt, Erfurt, Deutschland

Abstract aus Gau SY, Lee YH, Tsou HK, et al.: Patients with ankylosing spondylitis are associated with high risk of fibromyalgia: a nationwide population-based cohort study. Front Med (Lausanne) 2021;8:618594.

\section{Keywords}

Ankylosing spondylitis · Fibromyalgia · Cohort · Populationbased study · NHIRD

\footnotetext{
Abstract

Objectives: The main purpose of this retrospective cohort study was to provide an evaluation of Ankylosing spondylitis (AS) patients' fibromyalgia risk in different age and sex subgroups by analyzing large study samples.

Methods: Datasets from the National Taiwan Insurance Research Database (NHIRD) were retrieved in this retrospective cohort study. This study was approved by the Institutional Review Board of Chung Shan Medical University (IRB permit number CS15134). Within the Longitudinal Health Insurance Database (LHID), and the subset of NHIRD, we identified AS patients to explore the risk of further fibromyalgia. The exposure cohort included patients with newly-diagnosed AS (ICD-9-CM:720.0) during 2000-2013. After 1:4 age-sex matching and 1:2 propensity score matching, and adjusting potential confounders, individuals without AS were
}

identified as a comparison cohort. The adjusted hazard ratio of subsequent development of fibromyalgia in people with AS was evaluated. Further stratification analyses of different ages and genders were then undertaken to validate the results.

Results: In total, 17088 individuals were included in the present study, including 5,696 patients with AS and 11,392 individuals without AS. Respective incidence rates (per 1,000 person-months) of fibromyalgia was $0.52(95 \% \mathrm{Cl}, 0.46-0.59)$ in the AS cohort and $0.39(95 \% \mathrm{Cl}, 0.35-0.44)$ in the non-AS cohort. Compared with the non-AS cohort, aHR of developing fibromyalgia was $1.32(95 \% \mathrm{Cl}$, $1.12-1.55)$ in people with AS. This association was consistent in both statistical models of 1:4 age-sex matching and 1:2 propensity score matching.

Conclusion: Patients with AS were associated with a higher risk of fibromyalgia, especially those over 65 years old. In managing patients with AS, clinicians should be aware of this association, which could impact diagnosis, disease activity evaluation, and treatment. 


\section{Transfer in die Praxis}

\section{Hintergrund}

Die Hauptproblematik einer ankylosierenden Spondylitis (AS; auch bekannt als SpA für «Spondylitis ankylosans» - häufig beschrieben als Morbus Bechterew) ist der Schmerz. Bereits bei der Diagnosestellung und auch im weiteren Verlauf sind die Schmerzursachen jedoch nicht immer klar einer entzündlichen Genese zuzuordnen. Diese Ursachenklärung hätte jedoch unmittelbare, therapeutisch differierende Konsequenzen. Zudem ist bekannt, dass chronischentzündliche Erkrankungen wie die AS und z.B. auch die rheumatoide Arthritis oder der systemische Lupus erythematodes über die zentrale Schmerzverarbeitung eine sogenannte Zentralisation der Schmerzen und somit auch die Entwicklung einer Schmerzproblematik, einer Depression und auch einer Fibromyalgie begünstigen können. Somit wird zwar nach einer Fibromyalgie gehäuft anamnestisch gefragt, dennoch wird diese nur selten als Haupt- oder Nebendiagnose beschrieben. Studiendaten hierzu erscheinen widersprüchlich. Die Autoren dieser retrospektiven Kohortenstudie gehen daher der Frage nach, ob ein erhöhtes Risiko für die Entwicklung bzw. das Auftreten einer Fibromyalgie bei Patienten mit AS besteht und vergleichen die AS-Population mit einer ähnlichen Kontrollpopulation (Abb. 1).

\section{Studiendaten}

Nach klarer International Classification of Diseases (ICD)-basierter Krankheitszuordnung der AS und auch der Fibromyalgie wurden knapp 6000 AS-Patienten einer Kontrollpopulation von etwa 11000 anderweitig Erkrankten gegenübergestellt. Gemäß vorbekannter Literatur und mutmaßlichen Risikofaktoren für eine Fibromyalgie waren die beiden Kohorten gematcht in Bezug auf Alter, Geschlecht, Einkommen, Krankenhausaufenthalte, Stadt- oder Landleben, Komorbiditäten und Medikation. Wichtig hierbei ist, dass auch rheumatoide Arthritis und Depression neben inflammatorischen Problemen wie Diabetes mellitus, chronischen Leberund Nierenerkrankungen, chronisch-obstruktiver Lungenerkrankung (COPD) und Gicht als Diagnosen in beide Gruppen mit aufgenommen wurden. Der Beobachtungszeitraum der AS-Patienten betrug hierbei knapp 480 Patientenmonate.

\section{AS-Patienten haben ein höheres kumulatives Risiko für die Entwicklung einer Fibromyalgie}

In dieser großen AS-Population mit langer Beobachtungszeit zeigte sich ein deutlich erhöhtes Risiko für die Entwicklung einer Fibromyalgie im Vergleich zur Kontrollpopulation. Dies war bei Frauen im Vergleich zu den männlichen AS-Patienten noch einmal erhöht, wenn auch nicht statistisch signifikant. Gesteigert war dieses Risiko ebenfalls in der AS-Gruppe der Über-65-Jährigen. Bei der Medikation zeigte sich der Gebrauch von NSAR (nichtsteroidalen Antirheumatika) als Risikofaktor für das Entstehen einer Fibromyalgie. Dies ist mutmaßlich so zu interpretieren, dass entweder eine höhere inflammatorische Aktivität vorgelegen haben muss, die auch einen Risikofaktor per se darstellt, oder aber dass NSAR bei zeitgleichem Vorliegen von AS und Fibromyalgie keine ausreichende Wirkung haben können, aber dennoch weiterhin verordnet werden.

\section{Kommentar}

Fibromyalgie ist eine unerwünschte Diagnose - sowohl bei Ärzten als auch bei Betroffenen. Die Unterscheidung zu einem entzündlichen Schmerz erscheint zwar auf dem Papier recht einfach, in der Konsultation wird sie aber durch das Vorliegen sowohl entzündlicher als auch nichtentzündlicher Schmerzen erschwert. Diese können bei zunehmendem Alter des Betroffenen auch nicht immer klar von einer degenerativen Problematik abgegrenzt werden. Somit ist das Puzzle häufig nicht klar zu entziffern. Ein Mischbild aus allen 3 Komponenten erscheint realistisch. Die Erfahrung lehrt, dass eine klare anamnestische, klinische und bildgebende Evaluation zwar weiterhin der Grundstein zur Diagnosestellung ist, es dann jedoch wichtig ist, dem Patienten diese verschiedenen Komponenten darzulegen. Das braucht viel Zeit und Überzeugung. In einigen Fällen kann auch eine individuelle Teststrategie hilfreich zur Differenzierung sein: Ist eine anti-entzündliche Medikation wirklich noch hilfreich oder benötigt es eine konventionelle Analgesie? Ist doch eine Fibromyalgie im Vordergrund und kann somit eine multimodal-komplexe Schmerztherapie helfen? Diese Besprechung kann mit der Erklärung gepaart sein, dass eine chronisch-entzünd-
Abb. 1. Vergleich der kumulativen Wahrscheinlichkeit von Fibromyalgie bei Patienten mit und ohne AS unter verschiedenen Modellen. (A) Kumulative Wahrscheinlichkeit von Fibromyalgie bei AS-Patienten und 1:4 alters-/geschlechtsgematchten Kontrollen, die zum Indexdatum gefährdet waren. (B) Kumulative Wahrscheinlichkeit von Fibromyalgie bei ASPatienten und der 1:2 PSM-Kontrolle (aus: Front Med (Lausanne) 2021;8:618594). PSM = Propensity Score Matching.

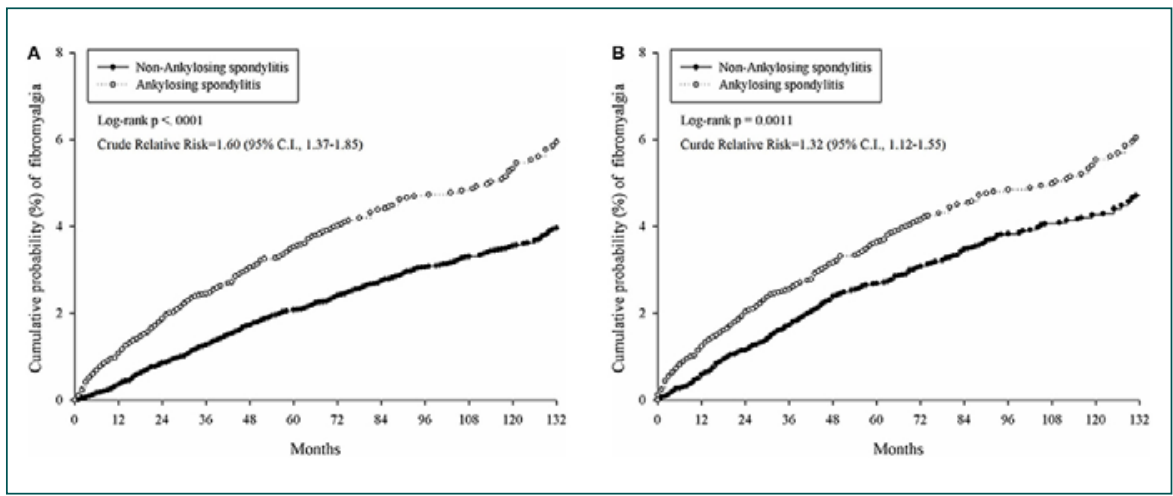


liche Erkrankung als unterliegende Erkrankung eine zentrale Schmerzverarbeitung beeinflusst und somit keine Schuldzuweisung und schon gar keine Stigmatisierung erfolgt.

Häufig erfolgt dennoch eine Eskalation der anti-entzündlichen Therapiestrategie. Wünschenswert wären jedoch mehr Konsultationszeit und der direkte Zugang zu einer multimodal-komplexen schmerztherapie.

\section{Fazitpunkte}

- AS und Fibromyalgie finden sich gehäuft - zeitgleich oder nacheinander.

- AS und Fibromyalgie treten häufiger bei Frauen auf.
- Die Differenzierung beider Entitäten ist zur Therapiekorrektur und Krankheitskontrolle notwendig.

- Eine spezifische interdisziplinäre Fibromyalgietherapie ist wünschenswert.

\section{Disclosure Statement}

Bezüglich des aktuellen Wissenstransfers bestehen keine Interessenkonflikte.

Korrespondenz an:

PD Dr. Sabine Adler, sabine.adler@helios-gesundheit.de 